\title{
Le privilège comme réponse à la concurrence commerciale étrangère : Nantes au début du $\mathrm{XVII}^{\mathrm{e}}$ siècle
}

\section{Guy Saupin}

\section{(2) OpenEdition}

1 Journals

\section{Édition électronique}

URL : https://journals.openedition.org/abpo/999

DOI : $10.4000 / a b p o .999$

ISBN : 978-2-7535-1517-8

ISSN : 2108-6443

\section{Éditeur}

Presses universitaires de Rennes

Édition imprimée

Date de publication : 20 avril 2010

Pagination : 75-90

ISBN : 978-2-7535-1146-0

ISSN : 0399-0826

\section{Référence électronique}

Guy Saupin, «Le privilège comme réponse à la concurrence commerciale étrangère : Nantes au début du xvII siècle ", Annales de Bretagne et des Pays de l'Ouest [En ligne], 117-1 | 2010, mis en ligne le 20 avril 2012, consulté le 21 septembre 2021. URL : http://journals.openedition.org/abpo/999; DOI : https://doi.org/10.4000/abpo.999

Ce document a été généré automatiquement le 21 septembre 2021.

(C) Presses universitaires de Rennes 


\title{
Le privilège comme réponse à la concurrence commerciale étrangère : Nantes au début du XVII siècle
}

\author{
Guy Saupin
}

1 Toute la première moitié du XVII siècle est caractérisée par un recours récurrent des marchands nantais au principe des privilèges de bourgeoisie pour contrer une concurrence commerciale étrangère jugée envahissante. Cette politique défensive, assez singulière dans son insistance dans l'histoire commerciale de la ville à l'époque moderne, témoigne d'un sentiment de déstabilisation du milieu marchand qui peine à s'adapter à une redistribution des cartes dans le grand cabotage européen. Une pression étrangère accrue ne semble pouvoir être contenue qu'en complétant les droits traditionnels de bourgeoisie afin de la retenir dans des limites juridiques bien définies. Dès le début du $\mathrm{XVII}^{\mathrm{e}}$ siècle, les Flamands, les Espagnols et les Portugais sont désignés comme les principaux envahisseurs. Dans les années 1610, deux projets de lettres patentes, pour l'adjonction au traditionnel privilège de bourgeoisie de l'obligation de passer par un marchand nantais pour toute transaction dans le commerce de gros et de détail, sont préparés au sein du consulat commercial. Cette réaction ne parvient pas à trouver sa traduction en termes de droit car elle ne rencontre pas le soutien des autorités municipales exprimant l'opinion de l'ensemble des élites urbaines.

2 L'enjeu est donc d'abord de replacer l'argumentaire de l'élite marchande dans la situation du commerce international nantais au début $d u x{ }^{\prime} I^{e}$ siècle afin de contextualiser au mieux les protections sollicitées par l'extension du privilège de bourgeoisie. La liste traditionnelle des privilèges, confirmée par Louis XIII à Paris en juin 1610, mentionne bien l'exigence de dix ans de résidence pour l'obtention des droits de bourgeoisie ${ }^{1}$, mais il n'existe pas de corps de bourgeoisie institué et contrôlé au sein de la communauté urbaine, même si cette dernière est désignée sous la forme canonique de l'ensemble des "nobles, bourgeois, manants et habitants de la ville et fauxbourgs ». À Nantes, comme dans bien d'autres villes du royaume, le droit d'habitanage, ne liant les privilèges qu'à une 
continuité de résidence, avait donc remplacé un pur droit de bourgeoisie ${ }^{2}$. Il s'agit ensuite de comprendre au nom de quelle logique l'assemblée municipale élargie, représentative de l'élite urbaine, s'est opposée à l'initiative marchande.

\section{La défense des marchands nantais par le droit de bourgeoisie : propositions de l'élite consulaire}

3 Le malaise économique et le réflexe juridique du recours aux privilèges commerciaux pour en sortir nous sont connus par deux documents : un projet de lettres patentes ${ }^{3}$ et une proposition de règlement en dix articles rédigé par les marchands ${ }^{4}$. Ces deux textes ne sont pas signés, mais comme la délibération de l'assemblée de ville parle des articles de "messieurs les marchands de Nantes », ces derniers ne peuvent venir que du consulat, tribunal de commerce servant aussi de représentation corporative de l'élite marchande nantaise, très lié la municipalité depuis leur fondation quasi conjointe en 1565.

4 La première rédaction préparatoire pour de futures lettres patentes n'est pas datée. Elle doit cependant correspondre à l'année 1610 puisqu'elle invoque au tout début la souveraineté d'Henri IV avant d'attribuer ensuite les décisions pratiques sollicitées à l'autorité de Louis XIII, suivant «l'advis de la royne régente, notre honorable dame et mère ». Il est donc possible que ce texte ait été formulé progressivement, de part et d'autre de l'assassinat d'Henri IV. L'argumentaire nantais débute habilement par une très belle déclaration d'intention mercantiliste qui prend soin de replacer Nantes et la Bretagne dans l'ensemble du royaume afin de souligner le potentiel économique considérable de ces deux entités pour travailler à la prospérité générale, doublement soutenue par le retour à la paix intérieure et par la politique de reconstruction du pouvoir royal.

«Scavoir faisons, comme l'ung des plus fortz nerfz et plus assuré support de l'estat de toute la monarchie consiste en l'abbondance de l'or et de l'argent qui fournist et suppedite aulx hommes toutes fortes necessitez en toutes saisons et occasions opportunes, laquelle abondance procedde pour la meilleure part de la négotiation de la marchandise et que l'oppulence et richesse des villes procede de ce traffic quy prend son accroissement des liberallitez graces et privillaiges desquelles elles sont décorées et ornées par leurs Roys, Princes et Seigneurs pour y rendre la marchandise plus affluente et abondante, et que pour ceste occasion Nos prédécesseurs Roys de France, ducz et seigneurs de nostre païs et duché de Bretaigne et nous à leur exemple et imitation avons toujours eu en très grande et singullière recommandation l'enrichissement et augmentation de nostre ville de Nantes audict pays et duché de Bretaigne, ville principalle et capitalle d'icelluy sittuée en l'embouchure de ceste belle et grande ripvière de Loyre, laquelle se coulent avecq une inffinité d'autres en ce grand océan qui l'avoisine et arrose chacun jour de son influance naturelle et là se communique à toutes les parties de cest univers, et que pour la boniffier davantaige et adjouter de nos graces et libérallitez à ce qu'elle a de bonté en sa naturelle situation, elle ait esté par nous et nos prédécesseurs décorée de beaux grands et amples privillaiges, immunitez, exemptions, franchises et libérallitez, en recognoissance desquelz nous et nos predecesseurs en ayons receu de grands secours lorsque le bien et la necessité de nos affaires l'ont requis comme nous en espérons recepvoir à l'advenir néantmoign de la part de nos amez et féaulx les maires et eschevins, nobles, bourgeois manants et habitans de la dite ville de Nantes. "

5 Cette rédaction stéréotypée permet toutefois d'éclairer sous deux angles complémentaires la stratégie collective de l'élite marchande nantaise au début du XVII ${ }^{\mathrm{e}}$ 
siècle. La référence à la logique contractuelle de l'attribution des privilèges dans un échange de libertés urbaines contre le loyalisme et le soutien au pouvoir royal doit évidemment escamoter la période de rébellion ligueuse (1589-1598), durant laquelle le milieu marchand fut le meilleur soutien au duc de Mercoeur, en la replaçant dans une période plus longue valorisant sa vigilance anti-protestante et la réconciliation de ses élites urbaines autour du trône. Cette reconstruction du loyalisme nantais se paie cependant par une nette réduction de l'influence du milieu marchand dans la vie municipale durant les quarante premières années $d u \mathrm{XVII}^{\mathrm{e}}$ siècle ${ }^{5}$, au profit du monde judiciaire dont les chefs ont incarné le loyalisme politique pendant la grande crise des années 1590.

6 Sous l'angle économique, ce plaidoyer, sous une certaine forme d'auto-satisfaction, révèle involontairement le principal danger qui guette l'élite marchande nantaise : l'enlisement dans la gestion préservée des courants d'échange passant obligatoirement par la ville, tout en laissant aux étrangers le soin de la relier aux nouveaux horizons. En insistant sur la fécondité de l'alliance entre les avantages naturels de sa situation de porte d'entrée et de sortie du plus grand réseau hydrographique du royaume et l'ensemble des privilèges concédés depuis trois siècles par les ducs de Bretagne et les rois de France, l'élite marchande nantaise laisse voir un manque de dynamisme créateur, une propension à défendre les acquis plutôt qu'à imaginer des alternatives de relance. Cette langueur pourrait bien être la traduction d'un plafonnement du système commercial en place dans lequel la croissance de certains secteurs comme celui du vin et des céréales masque mal la décrue du commerce du sel et surtout des toiles de lin et de chanvre. Socialement, elle signalerait également l'épuisement de l'apport dynamisant de l'immigration castillane, mal placée pour prendre l'initiative de nouveaux circuits ${ }^{6}$. Naccablons pas outre mesure cette élite marchande installée car c'est elle qui entreprend au même moment la conquête de l'armement à la grande pêche morutière au détriment de l'autonomie d'action de tous les petits ports du littoral, du Croisic aux Sables-d'olonne en passant par les havres de la baie de Bourgneuf. Ce n'est cependant que la captation d'un trafic existant pour lequel Nantes jouait déjà le rôle de grand marché de décharge au $\mathrm{XVI}^{\mathrm{e}}$ siècle $^{7}$ . L'augmentation des prises allait toutefois assurer une lente accumulation du capital qui allait s'avérer décisive dans l'invention du commerce avec les îles d'Amérique dans la seconde moitié du XVII ${ }^{\mathrm{e}}$ siècle.

7 L'argumentation commence habilement par une tentative de lier la défense des commerçants locaux et l'intérêt général du royaume, incarné par le pouvoir royal, en mettant en rapport le nécessaire soutien des revenus marchands et la capacité de répondre aux charges de la ville, relais de la puissance royale.

« Nous a esté faict plainte qu'ils (les marchands nantais) ne jouissent pas des fruitz et esmolluments entiers que ceste naturelle bonté de situation de leur ville avecq nos graces et libérallités leur debvroit apporter, ains y sont traversés et empeschés par une infinité de gens tant de Païs de Flandre, d'Espaigne, Portugal que aultres estrangers, lesquels y affluent et abbondent chacun jour et $\mathrm{y}$ font librement leur trafficq, les ungs y amenants de la marchandise et la revandans aulx estrangers sans laisser aucun gaign et profilt à faire aux habitants et domicilliaires de ladite ville, les autres y venants faire leur trafficq, amatz et achaptz de marchandises par eulx, leurs facteurs et associez et delà les transportent hors ladite ville et de notre Royaulme et font en effect tout ce que lesdits habitants et domicilliaires debvroient faire de trafficq et en emportent les gaigns et proffits qui leur debvroient justement appartenir pour supporter les frais et charges ordinaires de la dicte ville et nous apporter les secours tel le bien et soulaigement de nos affaires le requiert ${ }^{8}$. » 

Non pas qu'il n'y ait pas d'Espagnols à Nantes, car il s'agit de la communauté étrangère la plus importante depuis le milieu du xv $\mathrm{x}^{\mathrm{e}}$ siècle, mais la plupart d'entre eux - et surtout des plus puissants - sont à cette époque totalement intégrés ou en phase d'intégration dans la société nantaise ${ }^{9}$. Si les premières conventions avec les villes de Biscaye remontent au duc Jean Iv, le premier traité de commerce entre la Bretagne et la Castille fut signé par le duc Jean v en 1430. Cet accord, renouvelé cinq fois au $\mathrm{XV}^{\mathrm{e}}$ siècle, a établi à Nantes une « bourse et estappe coutumière » dirigée par un consul. Ceci a permis l'essor d'un puissant courant d'échanges reposant sur l'exportation des toiles de lin et de chanvre de tout l'Ouest français (Bretagne, Anjou, Maine et Poitou) comme de la quincaillerie et du papier des villes du Massif Central et, selon la conjoncture climatique, des céréales tirées du grenier à blé du littoral sud de la Bretagne, contre des importations de laines de Castille, de fer basque et de fruits méditerranéens, le tout faisant du marché castillan le premier horizon international nantais jusqu'au début $\mathrm{du} \mathrm{xvII}^{\mathrm{e}}$ siècle. Des échanges de privilèges ont spécialement lié les villes de Nantes et de Bilbao: les toiles bretonnes sorties du port ligérien n'acquittent pas de droit d'entrée dans celui du Nervión et réciproquement pour les laines de Castille ${ }^{10}$.

9 Le flux amenant les marchands basques et castillans - principalement de Vieille Castille autour de Burgos - fut ainsi régulier ${ }^{11}$ et l'insertion des grandes familles dans l'élite urbaine est une réalité ancienne, déjà mesurable à leur présence significative dans le Conseil des bourgeois de la ville dans la première moitié $\mathrm{du} \mathrm{xvI}^{\mathrm{e}}$ siècle, influence prolongée dans le nouvel échevinage installé en 1565, après des lettres patentes obtenues de François II en janvier 1560, non seulement par l'obtention de charges d'échevins, mais surtout par l'accès au poste de maire. La même situation caractérise le consulat commercial créé en 1565, tant les liens sont étroits entre les deux institutions. À partir de 1601, les premières archives conservées de la Casa de Contratación révèlent la prégnance des familles d'origine espagnole dans la direction de cette confrérie marchande gérant les chargements pour la péninsule Ibérique ${ }^{12}$.

Toutes ces raisons rendent donc surprenante l'insertion des Espagnols parmi la triade à contenir. Deux grandes explications semblent susceptibles de rendre compte de cette contradiction. Pour parvenir à une telle formulation, il faut bien admettre l'existence d'un clivage au sein même de la communauté hispanique nantaise opposant les familles anciennement implantées et les derniers arrivés jugés trop encombrants. Celui-ci ne peut être imputé à une brusque augmentation des arrivées car le passage entre les $\mathrm{XVI}^{\mathrm{e}}$ et XVII siècles correspond à une contraction numérique de cette communauté, au sein d'une tendance générale à la réduction. La moyenne annuelle des baptêmes passe ainsi de 4,3 pour la période 1571-1589, marquant l'apogée de la présence espagnole, à 2 pour les années 1590-1614, puis à 2,9 pour les années $1615-1650$ et enfin à 2,2 pour la période 1651-1690 ${ }^{13}$. La seule hypothèse possible renvoie alors vers une dégradation des profits révélant un certain affaiblissement du système d'échanges traditionnel. Les difficultés liées aux guerres de religion ne peuvent servir d'argument fondamental, même si l'entrée tardive mais radicale de la Bretagne, et surtout de Nantes, dans le conflit du côté de la Ligue en 1589 a doublement pris en tenaille le circuit commercial avec le contrôle des villes ligériennes d'amont par le parti royal et l'activisme de la course maritime rochelaise à l'embouche de la Loire. Le texte est rédigé en période de relance, après la paix de Vervins de 1598. Le malaise vient sans doute de plus loin et il est plus structurel que conjoncturel, ce qui renforce sa gravité ${ }^{14}$. Il semble bien que les marchands nantais et

Annales de Bretagne et des Pays de l'Ouest, 117-1 | 2010 
hispano-nantais, à l'image de la célèbre famille Ruiz, trop bien installés dans le réseau international d'échanges commandé par Burgos et privilégiant la côte cantabrique, le marché intérieur castillan et le grand cabotage européen vers les Pays-Bas, ait mal supporté le déclin de Burgos, la crise des Pays-Bas, surtout après la chute d'Anvers en 1585, mais surtout le basculement du centre de gravité de l'économie espagnole de la Vieille Castille en direction de l'Andalousie. Manquant d'esprit d'initiative pour tenter de s'infiltrer dans le monopole commercial en direction du marché américain en plein essor et de souplesse d'adaptation dans leurs relations marchandes avec les producteurs de toiles de l'Ouest au sein d'une concurrence accrue, ils se seraient fait progressivement damer le pion par leurs homologues malouins beaucoup plus inventifs sur les deux tableaux ${ }^{15}$.

La dénonciation de la concurrence portugaise et hollandaise semble beaucoup plus normale car il s'agit d'un phénomène relativement récent, amorcé surtout après 1598. L'immigration portugaise correspond au grand exode des familles marranes fuyant le renforcement de l'Inquisition. Elle secrète une tension économique, doublée d'une hostilité religieuse dans une ville très catholique comme Nantes, qui a connu sa phase la plus exacerbée dans la décennie $1630^{16}$. Le projet nantais parle de Flamands, mais il faut entendre derrière cette appellation principalement les marchands venus des ProvincesUnies beaucoup plus que leurs homologues des Pays-Bas méridionaux. L'essor d'une petite colonie marchande hollandaise a été plus lent, mais au total plus dangereux pour la communauté marchande indigène, ce qui explique que l'antagonisme ait connu sa phase la plus dure dans les années 1640 , relançant logiquement la même politique du recours au privilège comme système de protection ${ }^{17}$. Dans les années 1610, il s'agit donc d'un problème naissant, même si une émotion populaire anti-portugaise en 1603 avait déjà conduit le corps de ville à solliciter auprès du pouvoir royal l'expulsion de cet embryon étranger, permission refusée au nom de l'intérêt financier puisque les marranes se révélaient être les meilleurs introducteurs du métal argent espagnol en France, en partie par leurs réseaux de contrebande ${ }^{18}$.

Après avoir décrit l'injustice de la situation, le projet de lettres patentes prescrit le remède attendu :

«Le droict et privillaige de bourgeoysie pour eux seulz pouvoir achapter les marchandises lesquelles y sont amenées des autres royaumes et provinces et icelles revandre trocquer et retrocquer semblablement aux habitants desdits autres royaumes et provinces estrangères sans qu'il soit permis a aucuns forains non regnicolles de negotier et trafficquer soict par ventes ou achapts, trocquer et retrocquer avec autres forains et estrangers ${ }^{19}$. "

L'interdiction d'échanges directs entre étrangers et l'obligation de passer par l'intermédiaire des Nantais sont ainsi instituées comme nouveau privilège de bourgeoisie. Le texte laisse planer une certaine ambiguiité sur la définition du terme province, mais la désignation finale des «forains non régnicolles » laisse entendre que l'action est menée contre les seuls extérieurs au royaume et non pas contre les marchands non bretons. Peut-être cette apposition du terme de province à celui de royaume dans une perspective non française traduit-elle la crainte inspirée par la puissance émergente des ProvincesUnies, en guerre de sécession avec l'Espagne depuis 1581, mais signataires en 1609 d'une trêve dont le commerce français a tout à redouter. Cela vient s'ajouter à la paix conclue entre l'Espagne et l'Angleterre en 1604 et nul n'ignore que dans ces tractations diplomatiques l'accès des marchands nationaux au marché espagnol a été au cœur des plus vives discussions ${ }^{20}$. 
Suit la définition de l'accès au droit de bourgeoisie :

"Seront receuz en ladite bourgeoisie tous honnestes marchands et gens de bien et de bonne renommée trafficquants en ladite ville et forsbourgs natifz et originaires d'yceux et à semblable tous autres marchands non originaires pourveuz qu'ils y aient esté et soient domicilliaires demourantz et résidantz actuellement avecq leurs familles depuis les douze ans derniers. »

Les restrictions témoignent d'une bonne connaissance des réalités de la présence étrangère dans un port maritime et de l'importance des liens personnels dans la gestion des affaires à l'époque moderne. Les solutions sociétaires ou la constitution de réseaux d'échanges de services sur des circuits commerciaux se font principalement sur la confiance dans des partenaires dont on a pu éprouver la fidélité, d'où l'insistance sur la réputation sociale et la longueur de la résidence. En effet, nombre de marchands étrangers n'étaient là que de passage, pour quelques mois ou même quelques années, se logeant d'abord dans des auberges avant de se résoudre à prendre un loyer ${ }^{21}$. D'où la méfiance des marchands indigènes, toujours soupçonneux sur la solvabilité de partenaires mal cernés, sans vraiment de répondant immobilier. La tonalité se fait ici plus radicale, incluant même une rupture avec le paragraphe précédent puisque l'obligation des douze ans de séjour est étendue à tous les non-Nantais, y compris aux marchands français et même, chose plus surprenante, aux homologues bretons et pis encore du comté même de Nantes. La définition la plus stricte du horsain a ainsi prévalu.

Le passage à une gestion stricte de ce droit de bourgeoisie rénové aurait dû s'inscrire dans la création d'une nouvelle institution, appelant une collaboration étroite entre les marchands et la municipalité.

«Et presté le serment de maintenir et observer les pollices et règlement de ladite bourgeoisye, avons permis et permettons auxdits maire et eschevins de nommer, eslire par assemblée généralle quatre marchands de ladite bourgeoisye de deux ans en deux ans lesquels assisteront lesdits maire et eschevins pour recepvoir et faire enregistrer au greffe de ladite ville ceux qui seront receus audit privillaige de bourgeoisye et mesme pour juger avec lesdits maire et eschevins des contraventions qui pourront intervenir à cause de ladite bourgeoisye. »

Les archives municipales n'ont gardé nulle trace d'un document ressemblant de près ou de loin à un livre de bourgeoisie tenu en bonne et due forme comme il en a existé dans d'autres villes, principalement dans le nord et l'est de la France ${ }^{22}$, ni de procès-verbaux d'élections d'un bureau de contrôle ${ }^{23}$. En ce sens, Nantes ressemble plutôt à Paris où la bourgeoisie s'acquiert sans formalité2 ${ }^{24}$.

recours à l'arme des privilèges de bourgeoisie nantaise demandait effectivement de compléter et de préciser ces derniers en terme de concurrence commerciale. Dans les dix articles confirmés par Louis XIII en juin 1610, trois seulement étaient en rapport direct avec l'activité marchande : tenir une foire franche annuelle de 15 jours commençant à la fête de la Chandeleur (Jean v, 1407, Charles viII, 1493), avoir dans leurs maisons des poids, des mesures de blés, d'aulnage de draps et de toiles et élire et instituer des jaugeurs pour leur vérification (1407). Si l'exigence de dix ans de résidence pour l'accès des nonnaturalisés et des non-Nantais aux droits de bourgeoisie est bien mentionnée, il n'est par contre pas fait rappel de l'interdiction à tous les étrangers de vendre au détail du vin et des draps dans la ville de Nantes à moins d'y demeurer entièrement, accordée par le duc Jean IV le 30 septembre 1395. Il convenait donc de réunir clairement ces deux privilèges, en couvrant tous les produits et surtout en les étendant au commerce de gros. 
Tout en reprenant le principe d'obligation pour « toute personne foraine et estrangère non regnicolle » de ne contracter qu'avec un marchand bourgeois sous peine de 20 écus d'argent et confiscation des marchandises, le projet d'arrêt du conseil entend aussi réglementer le commerce de détail :

«[...] sans touteffois qu'ilz [les marchands bourgeois] puissent détailler sy non celles dont ils auront faict aprentissaige et auxquelles ils auront esté nouryz, apprins et faict exercice premier, comme aussy ne pourront tous forains et estrangers faire aucun détail et distribution par le menu de leurs marchandises, mesme aux habitants de ladite ville et forsbourgs, ains en demeurera et sera permis le détail seullement à ceux des habitants qui auront faict leur aprentissaige aux espèces des dits marchandises desquelles ils voudront faire le détail. »

20 En proposant un système protecteur pour le commerce de détail, l'élite marchande recherche le soutien de la masse des artisans et boutiquiers pour obtenir l'appui du corps de ville. Tout projet d'arrêt du conseil ne peut être présenté au gouverneur de la ville ou de la province, seuls tuteurs politiques à cette époque, qu'au nom de la municipalité, seule institution pouvant légitimement incarner le corporatisme bourgeois. Or, dans les années 1610, Nantes est encore en pleine transition quant au fonctionnement municipal. La tradition de convocation des assemblées générales ouvertes, qui s'était maintenue pendant la période des guerres de religion, retrouve un élan de courte durée après un premier affaiblissement sous la phase de pacification du règne d'Henri IV. Même si ce type d'assemblée consultative faisait surtout figure d'instance de cautionnement de la politique du corps de ville, les maitres artisans et boutiquiers pouvaient aisément faire masse. Il était donc stratégiquement habile pour le lobby marchand de rechercher le soutien des jurandes des arts et métiers, ne serait-ce que pour faire pression sur les maire et échevins. Mais là encore, les registres des délibérations de la ville ${ }^{25}$ ne mentionnent aucune assemblée convoquée sur ce sujet de 1610 à 1612, ce qui laisse augurer une certaine opposition à une politique réduisant l'autonomie d'action des marchands étrangers.

\section{Projet d'articles pour définir commercialement le droit de bourgeoisie et sa réfutation systématique par le corps de ville}

21 Un second texte de même tonalité nous est connu. Toujours non daté, il semble toutefois avoir été produit en 1613 puisqu'il est accompagné d'une réponse, portant cette date, de l'assemblée municipale qui en a débattu. Cette nouvelle mouture, présentée sous la forme de dix articles, a le mérite d'être plus précise et de lever certaines ambiguïtés antérieures autour de la définition du forain. Ce règlement s'intéresse à trois grands thèmes: la définition du bourgeois de Nantes, les privilèges commerciaux du droit de bourgeoisie, la préservation de certaines libertés des simples habitants. Comment cette initiative a-t-elle été reçue dans le corps de ville? Le débat a dû logiquement être ouvert dans le bureau servant formé du maire, des six échevins ${ }^{26}$ et du procureur du roi syndic et peut-être se prolonger dans le grand bureau municipal formé de tous les maires et échevins, servants comme anciens, selon la procédure coutumière, mais l'absence de registre de délibérations de mai 1612 à juin 1615 nous empêche de le vérifier.

22 La pièce d'archive isolée conservée fait état d'une structure plus élargie, à mi-chemin entre le grand bureau et l'assemblée générale des nobles, bourgeois et manants de la ville 
et faubourgs. C'est ce que nous indique le tout début du texte : «L'advis de messieurs les députés pour voir les articles proposés par messieurs les marchands de Nantes [...]. » La formule caractérise l'ouverture du grand bureau aux députés des trois grands corps traditionnels qu'étaient l'Église de Nantes, représentée généralement par deux chanoines de la cathédrale Saint-Pierre ou de la collégiale Notre-Dame, la chambre des comptes de Bretagne qui déléguait deux de ses officiers et la cour de la sénéchaussée et du présidial de Nantes qui procédait de même, ce siège de justice royale bénéficiant en plus du privilège de présider l'assemblée par la voie de son sénéchal ou d'un autre officier supérieur par ordre hiérarchique. L'invitation des gouverneurs de ville et de la province était de droit, mais leur présence effective dépendait de leurs déplacements géographiques entre la cour, la ville, leurs seigneuries et les champs de bataille. D'autres corps urbains pouvaient être appelés selon le thème discuté : ainsi les capitaines de milice bourgeoise en temps d'insécurité ou les juge et consuls des marchands pour les questions commerciales ${ }^{27}$. La pure logique voudrait donc qu'ils aient été appelés, mais le résultat de la délibération permet d'en douter. Pour justifier sa réfutation systématique de tous les articles, la municipalité a pris soin de rédiger une introduction qui met l'accent sur trois inconvénients : les conséquences négatives pour l'activité commerciale du port de Nantes, les retombées problématiques sur les finances municipales et la mise en cause de l'identité bretonne dans le partage des privilèges. Sa conclusion est sans appel : il n'est nul besoin de modifier la charte des privilèges traditionnels confirmés par Louis XIII à son avènement.

Il est ainsi éclairant de comparer point par point les deux logiques qui se sont affrontées. Les deux premiers articles définissent le droit de bourgeoisie, lié à la naissance ou à une certaine durée de résidence en continu.

«Que pour acquérir le droict de bourgeoisie et se pouvoir dire bourgeois de ladite ville, il s'entend estre né dans ladite ville et forsbougs banlieue ou comté et faire sa résidance dans ladite ville ou bien estant forain qu'il y ait faict sa résidance comme bon habitant durant la période de dix ans entiers et à commencer du depuis qu'ils auront faict leur déclaration au corps de ville qu'ils entendent s'y habituer.»

La même définition est appliquée aux étrangers, à partir de l'enregistrement de leurs lettres de naturalité. La période classique de dix ans est ici confirmée et il semble donc que la mention de douze ans dans le premier projet soit liée à une méconnaissance de la charte des privilèges, ce qui renvoie vers une souplesse de son application ${ }^{28}$. La volonté de passer à une gestion institutionnelle rigoureuse du droit de bourgeoisie est aussi réaffirmée puisque ce délai de dix ans ne pourrait être pris en compte qu'à partir d'un acte administratif précis : l'enregistrement au bureau de ville pour les forains et lettres de naturalité à la chambre des comptes pour les étrangers. Mais la principale innovation tient dans la distinction opérée entre les gens du comté nantais et tous les autres régnicoles, y compris les habitants des autres évêchés bretons, tous rangés sous l'appellation de forains. Cette distinction est hautement significative du caractère très agressif de la démarche quand on sait l'importance des échanges entre Nantes et la Bretagne, premier marché pour l'écoulement du sel et des vins de Loire et pour l'approvisionnement en céréales et toiles de lin et chanvre ${ }^{29}$. Ce durcissement se lit également dans l'élévation de l'amende à 200 livres tournois.

Le rejet de l'initiative marchande par l'oligarchie municipale est sans appel. Toute l'argumentation est contrée sur quatre points principaux. Premièrement, les dangers de réciprocité de cette politique ultra-protectionniste fondée sur le privilège d'exercice du commerce sont directement dénoncés comme contre-performants du point de vue du 
dynamisme économique de la ville: "[...] la qualité de bourgeoisie et droict de bourgeoisie aux habitants seuls de la ville et forsbourgs de Nantes seroit plus nuisible que profitable d'aultant que seroit exclure les alliances et commerce avec les aultres villes de ce royaulme et principallement des voisins dont il y en a plusieurs et esloigner tous les marchands non seulement estrangers mais encore de toutes les autres proches d'icelle [... ]$^{30}$.» Toutes les échelles du commerce sont envisagées, tant régionale que nationale et surtout internationale. Le terme d'alliance utilisé dans la rédaction renvoie plus aux circuits commerciaux résultant des liens personnels établis dans les réseaux marchands qu'à des traités négociés, car il n'existait pas d'équivalent à l'accord particulier liant Nantes et Bilbao.

Le second reproche concerne la gestion de l'ensemble de la police urbaine dans la mesure où ce règlement conduirait "à donner lieu à tous les habitans de touttes qualités de s'exempter des charges de la ville avant d'avoir acquis le droit de bourgeoisie [...]». Les charges dont il est question concernent principalement les taxations exceptionnelles par chef de famille selon la richesse estimée puisque l'ensemble des habitants est exempté des fouages depuis le duc Jean III en 1332, mais surtout Jean V en 1407. Celles-ci se sont multipliées au temps des guerres civiles et les exigences fiscales répétées du roi Henri IV dans la phase de restauration du royaume pouvaient légitimement inquiéter la municipalité, même si la priorité était de répondre en alliant l'utilisation des octrois et le recours aux emprunts ${ }^{31}$. La résurgence de l'effervescence nobiliaire durant la régence de Marie de Médicis pouvait aussi faire craindre des risques de prises d'armes et donc des charges de mise en défense pour une ville qui avait beaucoup à se faire pardonner en effaçant par un loyalisme indéfectible son mauvais choix ligueur, dans une province dont le gouverneur était le duc de Vendôme, demi-frère du roi et gendre du duc de Mercoeur, propriétaire de la baronnie d'Ancenis depuis 1599 .

La troisième opposition soulignait la contradiction fondamentale entre l'affirmation de la volonté d'intégration de tous les acteurs les plus dynamiques du commerce à l'intérieur de la bourgeoisie nantaise et l'exigence de dix ans de résidence pour vérification de bonne conduite. Pour l'oligarchie urbaine, cette longueur excessive n'aboutirait qu'à la désertification de la place nantaise car aucun immigré entreprenant ne saurait accepter pendant si longtemps une telle limitation de ses initiatives. Le texte constate que ce serait " oster tout moien à ceux qui aspireroient de faire aulcun traffic et par conséquent les obliger à s'en bannir eux mesmes ». La dernière critique porte sur la distinction entre le comté nantais et le reste de la Bretagne. «Oultre d'apeller forains les habitans des autres villes de la Province seroict diviser de nom et deffaict ladite ville de Nantes d'avec touttes les autres de ladite Province et non seulement les convier mais encore les obliger d'en faire le semblable et par conséquent nous randre aussi à eulx forains et faire naistre une espidémie qui tourneroit en animosité entrelles. » Au-delà du risque d'entrer dans le cercle vicieux de l'agressivité commerciale, les députés des corps ne peuvent que s'inquiéter des conséquences négatives sur la question de la défense de l'identité bretonne et des privilèges concédés par la monarchie dans l'édit d'union de $1532^{32}$, ensemble de franchises et de libertés dont les États provinciaux ont la garde et dont la capacité protectrice pourrait être altérée par des dissensions internes au tiers état, envenimées par cette opposition entre Nantes et les villes des autres évêchés ${ }^{33}$. Peut-être faut-il voir ici, dans cette divergence d'appréciation de l'identité bretonne, le sentiment d'être mal protégé par des États totalement dominés par les propriétaires fonciers structurés dans les systèmes clientélistes des grandes familles nobiliaires, système dans 
lequel la ville de Nantes a bien du mal à faire entendre sa voix, surtout lorsque sa délégation est marchande comme cela arrivait dans la seconde moitié du XvI ${ }^{\mathrm{e}}$ siècle ${ }^{34}$. La priorité donnée à la surtaxation de la consommation du vin au détail pour financer la défense des privilèges bretons pouvait inquiéter le monde marchand, mais elle n'a pas eu d'effet négatif avant le milieu du $\mathrm{xVII}^{\mathrm{e}}$ siècle. C'était par ailleurs compensé par l'atténuation des taxes sur les exportations de grains et de toiles.

Les articles 3 et 4 qui tendent placer le marchand bourgeois comme intermédiaire obligé dans toutes les transactions sont critiqués au nom d'une logique libérale du grand commerce et de leurs conséquences néfastes sur le marché du travail et sur les revenus de la ville et du roi. Le monopole bourgeois est d'abord dénoncé pour ses effets négatifs sur l'évolution des prix.

«Les marchands bourgeois ayant eux seuls la liberté d'achepter en gros les marchandises qui viennent par eau et par terre pourroient user de grandes rigueurs a l'endroit des marchands forains et imposer a leurs marchandises tel prix que bon leur semblera qui seroit les chasser et leur donner occasion de ny retourner plus, et puis pour la revente aux forains et habitans il seroit en leur liberté de revendre a prix excessif et a volonté, ce qui seroit grandement préjudiciable aux debvoirs du Roy et au Publicq.»

Cette défense de la libre concurrence pour la régulation des prix pourrait sembler curieuse dans un temps où les principes de l'économie morale définis à partir des valeurs chrétiennes restent prépondérants. L'apparente contradiction disparait en considérant le type de commerce envisagé et la nature de l'institution exprimant ce point de vue. Il s'agit du commerce de gros pour lequel les acteurs ont toujours refusé un encadrement corporatif à la différence du commerce de détail largement réglementé par les confréries de métiers. Dans l'idéologie de l'économie dirigée charitable, il ne peut être question de laisser la régulation entre les mains d'un des acteurs économiques car elle ne peut relever que des institutions détenant légitimement la responsabilité de protection de l'ensemble de la communauté urbaine, en premier lieu le corps de ville, mais aussi le pouvoir royal par le biais de son administration ${ }^{35}$. Il n'est donc pas étonnant qu'une assemblée corporative municipale dominée par les officiers de justice ne veuille pas laisser échapper cette prérogative. L'intervention de l'autorité publique sur le seul marché de consommation n'est d'ailleurs conçu qu'en cas de crise, afin de rétablir par une aide financière à l'importation un meilleur équilibre entre l'offre et la demande. L'analyse du fonctionnement de l'échevinage nantais durant toute la première moitié du XvII ${ }^{\mathrm{e}}$ siècle montre d'ailleurs la grande différence de rigueur entre le traitement des détaillants et des grossistes, des boulangers et des marchands blatiers pour le marché céréalier ${ }^{36}$, de loin le plus sensible.

Le reste de l'argumentation prend un tour assez démagogique puisqu'y est agité le risque de tensions sociales par privation d'emploi, crispation dont les pouvoirs publics cherchent à se prévenir le mieux possible.

«[...] en outre, on osterait les moyens de vivre à grand nombre de pauvres habitans qu'à présent ont liberté d'achapter des marchands forains pour en faire la revente, et toutefois ces particuliers sont considérables dans le publicq de la ville parce qu'ils aydent a porter les charges d'icelles [...]. »

31 Même en se souvenant que l'essentiel des ressources municipales vient des octrois urbains pesant sur la consommation de l'ensemble des habitants, et tout spécialement des boissons, il est douteux que la catégorie identifiée soit vraiment essentielle pour les finances municipales. Tout dépend du sens donné à l'expression « pauvres habitants ». S'il 
s'agit de l'ensemble du secteur boutiquier, on ne peut qu'être d'accord; s'il s'agit simplement des regrattiers, comme le vocabulaire y invite, on ne peut que trouver l'appréciation exagérée. En replaçant les questions fiscales au cœur de leur argumentaire, les maires et échevins et députés des corps, qui ont bien conscience de plaider devant le pouvoir royal, rappellent avec opportunité combien, pour celui-ci, l'appréhension de son rapport avec les villes fut avant tout fiscale ${ }^{37}$. Toute ville bonne payeuse, tant pour les taxes que pour le placement des rentes royales, était assurée de conserver son catalogue de privilèges.

L'ensemble des articles allant de 5 à 8 , où le projet cherchait à garantir un certain nombre de libertés aux habitants tant pour leur approvisionnement familial que pour la vente des produits de leurs propriétés, est rapidement critiqué comme non avenu puisque ces prescriptions relèvent du droit commun, sur lequel tout ajout ne pourrait qu'offrir opportunité de restriction. Il en est même des privilèges des foires et marchés. La conclusion est de s'en tenir à la charte traditionnelle des privilèges. L'article 9, qui entendait réserver la possession des poids et mesures aux seuls marchands bourgeois, est totalement récusé puisque ce privilège est acquis à l'ensemble des habitants depuis le duc Jean $\mathrm{v}$ en 1407, sous le contrôle du corps de ville. L'idée d'imposer le mariage avec une Nantaise à tout marchand résident est dénoncée comme saugrenue et en opposition à la réalité sociale de l'exercice du grand commerce observable dans la ville même. Tout l'arsenal d'amendes et de peines prévues dans le dernier article n'a pas lieu d'être puisque l'ensemble du dispositif a été invalidé, la sanction des fraudes dans le cadre de l'organisation classique du commerce étant renvoyée aux règles et aux instances de police existantes, c'est-à-dire le tribunal où sont associés le prévôt et les maire et échevins depuis le compromis passé en $1581^{38}$.

Les équilibres sociologiques dans le corps de ville ne sauraient suffire à expliquer pourquoi ce dernier a bloqué dès le départ la tentative des marchands nantais, sans doute élaborée au sein du consulat du commerce, de se protéger d'une concurrence étrangère jugée aggravée, en renforçant les privilèges traditionnels de bourgeoisie. Peut-être l'une des causes tient-elle dans le caractère très exagéré de la menace, jusqu'à en brouiller la légitimité ? Plus certainement, on voit bien combien les états sociaux supérieurs, très liés dans leurs revenus à l'agriculture commerciale, ne veulent pas se laisser enfermer dans un face-à-face avec une catégorie marchande en situation de monopole, sur les marchés intérieur et extérieur.

Il est ainsi plaisant de voir un corps de ville peuplé de juristes donner un cours d'économie politique à son élite marchande. Ces discordances témoignent surtout des difficultés d'adaptation d'une ville portuaire française à une économie commerciale mondialisée, phénomène dans lequel elle n'a alors joué qu'un rôle de relais en étant intégrée dans des circuits dominés par des centres d'impulsion extérieurs. Alors que le renforcement d'une communauté portugaise a produit d'autres formes d'agressivité, la même tentative de résistance juridique a caractérisé les relations avec les Hollandais dans les années 1640 . Toutefois, dans cette seconde phase, le jeu était double puisque les plus entreprenants des marchands nantais posaient les premiers jalons de l'organisation du commerce de droiture avec les îles d'Amérique dans le cadre de la colonisation française. Cette nouvelle dynamique commerciale les a incités à préférer assez vite la collaboration 
économique à la guerre des privilèges, dans la mesure où existait dorénavant un nouvel horizon spéculatif prometteur.

\section{NOTES DE FIN}

1. Arch. mun. de Nantes, AA3, f० 28, 29. «Que les estrangers qui ne sont nationalizés et asses qui n'auroient esté par l'espasse de dix ans habitués en ladicte ville et fauxbourgs ne pourront jouir desdits privilleiges et droits de bourgeoisie concédez ausdicts bourgeois ». Avant son enregistrement, la chambre des comptes a vérifié l'exacte correspondance avec les lettres antérieures, et plus spécialement celles du 3 décembre 1580, celles concédés par Henri IV au duc de Mercoeur lors de sa reddition en mars 1598 et reprises le 23 mai 1598.

2. Bonin, Pierre, Bourgeois, bourgeoisie et habitanage dans les villes du Languedoc sous l'Ancien Régime, Aix-en-Provence, PU Aix-Marseille, 2005, p. 39-45, 193-197.

3. Arch. mun. de Nantes, AA 15, fo 5 et 6 . Il existe deux copies différentes du même projet. 4. Arch. mun. de Nantes, AA $15, f^{\circ} 1$ et 2.

5. SAUPIN, Guy, Nantes au XVII ${ }^{e}$ siècle. Vie politique et société urbaine, 1598-1720, Rennes, PUR, 1996, p. 153-165. La décennie 1610 est mauvaise pour la représentation marchande puisque les élections aux deux postes renouvelables n'ont promu que cinq marchands contre onze juristes, trois officiers de finance et un médecin, soit le quart de l'échevinage alors que la proportion globale pour la période 1598-1639 atteint quand même 32,6 \%. 6. TANGUY, Jean, Le Commerce nantais à la fin $d u X I^{e}$ et au début du XVII siècle, Thèse de $3^{\mathrm{e}}$ cycle, dact., université de Rennes, 1967, p. 37-138 (vin-sel-blé), 163-181 (textiles), 233-234 (bilan). Collins, James B., La Bretagne dans l'État royal. Classes sociales, États provinciaux et ordre public de l'Édit d'Union à la révolte des Bonnets rouges, Rennes, PUR, 2006, p. 59-80.

7. Ibidem, p. 147-162.

8. Arch. mun. de Nantes, AA $15, f^{\circ} 5$ et 6.

9. CROIX, Alain (dir.), Nantais venus d'ailleurs. Histoire des étrangers à Nantes des origines à nos jours, Rennes, PUR, 2007, p. 26-35.

10. ZABALA URIARTE, Aingeru, « Les relations maritimo-commerciales entre Bilbao et certains ports du Nord-Ouest français (1610-1620) », dans PRIOTTI, Jean-Philippe et SAUPIN, Guy (dir.), Le Commerce atlantique franco-espagnol. Acteurs, négoces et ports (XV'e-XVIII siècles), Rennes, PUR, 2008, p. 183-213.

11. CRoIX, Alain (dir.), Nantais venus d'ailleurs..., op. cit., p. 27. En reprenant l'ensemble des lettres de naturalité, l'auteur souligne l'importance du second $\mathrm{XVI}^{\mathrm{e}}$ siècle (surtout après la paix de 1559) avec 45 lettres dont 27 résidences nantaises sûres et 18 présumées, devant le premier $\mathrm{XVI}^{\mathrm{e}}$ siècle avec 17 lettres, dont 10 sûres et 7 probables. Ceci contraste avec un effondrement du XVII ${ }^{\mathrm{e}}$ siècle, avec respectivement 12 lettres (6 et 6) pour l'ensemble du siècle.

12. Arch. mun. de Nantes, HH 194. Jeulin, Paul, « Aperçus sur la Contratación de Nantes, 1520 environ-1733 », Annales de Bretagne, tome XL, 1932-1933, p. 284-331, 457-505. Les familles hispano-nantaises ne sont pas les plus nombreuses au début du XVII ${ }^{\mathrm{e}}$ siècle, mais 
elles conservèrent une certaine prépondérance. Cela se voit à l'évolution de l'équilibre des deux groupes dans le rang de classement sur la liste de 1601: 5 familles « espagnoles » et 5 françaises dans les 10 premiers, 12 et 18 dans les trente premiers, 15 et 25 dans les quarante premiers. Cela se marque surtout pour l'octroi du poste de consul où seule la famille Poullain peut rivaliser avec la notoriété de De Bourgues, Despinoze et SantoDomingo.

13. BREJEON Jean-Joël, LA VILLETANET, Yves de, Le judaïsme à Nantes du XVI $I^{e}$ à la fin du XVII siècle, mémoire de maîtrise, dir. Yves Durand, université de Nantes, 1970.

14. CRoIX, Alain, L'Âge d'or de la Bretagne, 1532-1675, Rennes, Ouest-France université, 1993, p. 189-190.

15. PRIOTTI, Jean-Philippe, «Conflits marchands et intégration économique (Bretagne, Castille et Andalousie, 1560-1580) », dans PRIOTTI, Jean-Philippe et SAUPIN, Guy (dir.), Le Commerce atlantique franco-espagnol..., op. cit., p. 73-99.

16. SAUPIN, Guy, « Un mouvement de xénophobie anti-portugaise à Nantes dans les années 1630 », Poussou, Jean-Pierre et al. (dir.), Monarchies, noblesses et diplomaties européennes (Mélanges Labourdette), Paris, PU Paris Sorbonne, 2005, p. 49-60.

17. Arch. mun. de Nantes, AA 15, f ${ }^{\circ}$ 7-10. SÉE, Henri, « Un document sur le commerce des Hollandais à Nantes en 1645 », Economisch Historisch Jaarboek, 1926, p. 125-134. CRoIX, Alain (dir.), Nantais..., op. cit., p. 70-72.

18. ZABALA URIARTE, Aingeru, « La communauté marchande portugaise dans les ports de Bilbao et Saint-Sébastien au début du XVII e siècle », dans SAUPIN, Guy (dir.), Villes atlantiques dans l'Europe occidentale du Moyen Âge au XXe siècle, Rennes, PUR, 2006, p. 179-203. 19. Arch. mun. de Nantes, AA 15, fo 5 et 6.

20. GELABERT, Juan E., « Entre « arrêt général » et « libre trafic » : les relations commerciales entre la France et l'Espagne de 1598 à 1609 », dans PRIOTTI, Jean-Philippe et SAUPIN, Guy (dir.), Le Commerce atlantique franco-espagnol..., op. cit., p. 161-182.

21. BotTin, Jacques, CALABI, Donatella (dir.), Les Étrangers dans la ville, Paris, Éd. MSH, 1999, p. 1-9, 281-298 (Rouen), 329-342 (Bordeaux).

22. Voir la très riche recension bibliographique dressée par Pierre BONIN, Bourgeois..., op. cit., p. 29, 43-44.

23. On voit mal comment le corps de ville aurait pu admettre cette prétention du milieu marchand à garantir la bourgeoisie, car ce n'était pas une catégorie supérieure dans la hiérarchie des états au sein de l'élite urbaine, surtout dans cette période où il était politiquement au purgatoire afin de se laver de son basculement majoritaire dans la rébellion dans les années 1590 .

24. Descimon, Robert, « Bourgeois de Paris, les migrations sociales d'un privilège », dans $C$ HARLE, Christophe (dir.), Histoire sociale, histoire globale ?, Paris, Éd. MSH, 1993, p. 173-182. C ORCIA, J. di, « Bourg, Bourgeois, Bourgeois de Paris from the Eleventh to the Eighteenth Century » dans The Journal of Modern History, vol. 50, juin 1978, p. 207-233.

25. Arch. mun. de Nantes, BB 27. Du 9 juillet 1609 au 4 mai 1612. La vérification n'a rien donné. Malheureusement, il nous manque toutes les délibérations de mai 1612 à juin 1615. BB 28 (16 juin 1615-30 novembre 1617).

26. La date imprécise fait hésiter entre la mairie dirigée par Jean Blanchard, procureur du roi au présidial (1611-1613) et celle de Louis Charrette, sénéchal de Nantes (1613-1615). Dans les deux cas, nous n'avons qu'un seul marchand isolé dans un bureau dominé par les juristes, surtout officiers royaux.

27. SAUPIN, Guy, Nantes au XVII siècle..., op. cit., p. 109-116. 
28. L'hésitation peut s'expliquer par la variation des exigences théoriques dans les autres grandes villes marchandes du royaume, depuis 12 ans à Marseille, Lyon, Rouen depuis 1630, jusqu'aux 10 ans de Narbonne, cinq ans de Toulouse, deux ans de Bordeaux et un an et un jour de Paris. Il convient toutefois de différencier les règles et la pratique puisqu'à Toulouse la seule résidence suffisait largement, au moins la moitié des habitants négligeant de se faire inscrire au greffe du capitoulat. Voir les exemples contradictoires et relatifs relevés par Pierre Bonin, Bourgeois, bourgeoisie..., op. cit., p. 54-58.

29. TANGUY, Jean, Le Commerce nantais..., op. cit., p. 274-298.

30. Arch. mun. de Nantes, AA 15, fo 3 et 4.

31. SAUPIN, Guy, « Les octrois de Nantes de la création de la municipalité à 1732 », dans H AUDRÈRE, Philippe (dir.), Pour une histoire sociale des villes (Mélanges J. Maillard), Rennes, PUR, 2006, p. 43-57.

32. CroIX, Alain, L'Âge d'or..., op. cit., p. 13-28.

33. Collins, James B., La Bretagne dans l'État royal..., op. cit., p. 191-207.

34. Ibidem, p. 38-47, 156-164.

35. GUIGNET, Philippe, Le Pouvoir dans la ville au XVIII siècle. Pratiques politiques, notabilité et éthique sociale de part et d'autre de la frontière franco-belge, Paris, Éd. EHESS, 1990. L'auteur est celui qui a posé avec la plus grande pertinence la question de l'idéologie municipale sous l'Ancien Régime pour les villes des anciens Pays-Bas en caractérisant le modèle hispano-tridentin. La comparaison avec l'ensemble des villes françaises au XVII ${ }^{\mathrm{e}}$ siècle n'a pas été suffisamment développée. Les recherches de Yann LIGNEREUX sur Lyon sont cependant un début de réponse. Voir : Lyon et le Roi. De la « bonne ville » à l'absolutisme municipal (1594-1654), Seyssel, Champvallon, 2003, p. 583-590, 681-693.

36. CROIX, Alain, La Bretagne aux $16^{e}$ et $17^{e}$ siècles. La vie, la mort, la foi, Paris, Maloine, 1981, tome 1, p. 393-419.

37. BeIK, William, Urban Protest in $17^{\text {th }}$ Century France. The Culture of Retribution, Cambridge, CUP, 2004 (1997). Coluns, James, The State in Early Modern France, Cambridge, CUP, 2006, (1995).

38. SAUPIN, Guy, Nantes au XVII siècle..., op. cit., p. 50-52.

\section{RÉSUMÉS}

La décennie 1610 est marquée par une tentative de défense juridique des élites marchandes nantaises pour contrer une augmentation de la concurrence étrangère (Portugais, Flamands et Hollandais). L'élite qui fournit les juges du consulat commercial essaie de se prémunir en recourant aux privilèges de bourgeoisie. Des projets d'articles pour leur donner un contenu commercial sont ainsi rédigés, mais rejetés intégralement par le corps de ville et son assemblée générale corporative consultative. Ces tentatives de réponse juridique à un problème économique témoignent des difficultés d'adaptation d'une ville portuaire atlantique française aux mutations d'une économie commerciale mondialisée dans laquelle le centre de gravité européen glisse lentement du sud vers le nord. Le paradoxe naît d'une opposition d'une municipalité dominée par les juristes à un réflexe défensif juridique porté par le milieu marchand.

The 1610 decade is marked in Nantes by a legal attempt from the merchant elite to thwart a growth of the foreign competition (Portuguese, Flemish, Dutch). The elite supplying the bench of the merchant consulate tries to protect itself by using the privileges of citizenship. So, articles 
planned to give the latter a mercantile definition are written but are completely rejected by the town council and its general consultative assembly. These attempts at a legal reply to economic problem show how difficult it is to adapt a French Atlantic sea-port to the changes of a global merchant economy in which the European centre of gravity slowly slips from south to north. The paradox comes from an opposition of a corporation dominated by legal experts to a legal defensive reaction supported by the merchant circle.

INDEX

Thèmes : Nantes

Index chronologique : XVIIe siècle

\section{AUTEUR}

\section{GUY SAUPIN}

Professeur d'histoire moderne Université de Nantes Centre de Recherche en Histoire Internationale et Atlantique - EA 1163 